\title{
Heat Flow in a Right Circular Cylinder With Internal Heat Generation - Applications to the Determination of Thermal Conductivity
}

\author{
D. R. Flynn \\ Institute for Applied Technology, National Bureau of Standards, Washington, D.C. 20234
}

(June 8, 1967)

\begin{abstract}
Expressions are developed which permit calculation of the temperature-dependent thermal conductivity of a cylindrical specimen in which heat is generated internally, e.g., by radioactive decay. The information needed consists of the experimentally determined temperature distributions on the surfaces of the cylinder, the heat flow through a central circular area at one end of the cylinder, and the rate of internal heat generation (which in general may be position-dependent). Numerical coefficients are tabulated for the case of uniform internal heat generation. The application of this calculation procedure to published methods of thermal conductivity determination is shown and an example is given.
\end{abstract}

Key Words: Heat conduction, heat generation, heat transfer, neutron absorption, radioactive decay, thermal conductivity.

\section{Introduction}

Subsequent to the publication of a paper $[1]^{1}$ in which a mathematical analysis of heat flow in a right circular cylinder with arbitrary temperature boundary conditions was made, we were requested by $\mathrm{D}$. L. McElroy of the Oak Ridge National Laboratory to consider the problem of determining the thermal conductivity of a cylinder in which internal heat generation is also present. Since most methods of experimentally measuring thermal conductivity are not readily applicable to self-heating specimens, and since there is a real need for determining the thermal properties of radioactive materials or of materials which are being irradiated, we felt the present analysis to be of sufficient interest to warrant publication even in the absence of any experimental data.

\section{Mathematical Development}

Consider a homogeneous, isotropic, opaque, solid right circular cylinder of radius, $b$, and thickness, $l$, with position-dependent heat generation, $W(\mathbf{r})$, per unit volume per unit time, where $\mathbf{r}$ is the position vector. In general, the thermal conductivity of the cylinder material may vary with temperature. The steady-state heat flow equation in cylindrical coordinates is

$$
\begin{array}{r}
\nabla \cdot(k \nabla v)=\frac{1}{r} \frac{\partial}{\partial r}\left(k(v) r \frac{\partial v}{\partial r}\right)+\frac{\partial}{\partial z}\left(k(v) \frac{\partial v}{\partial z}\right) \\
+\frac{1}{r^{2}} \frac{\partial}{\partial \theta}\left(k(v) \frac{\partial v}{\partial \theta}\right)=-W(\mathbf{r})
\end{array}
$$

\footnotetext{
' Figures in brackets indicate the literature references at the end of this paper.
}

where temperature above an arbitrary datum is denoted by the symbol, $v$, the temperature-dependent thermal conductivity by $k(v)$, and the radial, longitudinal, and angular coordinates by $r, z$, and $\theta$, respectively. Equation (1) can be reduced to a simpler form by introduction of a new variable, $u$, defined by the relation

$$
u=\frac{1}{k^{*}} \int_{0}^{v} k\left(v^{\prime}\right) d v^{\prime}
$$

where $k^{*}$ is the value of $k(v)$ at $v=0$. Making the substitution (2), (1) reduces to

$$
\nabla^{2} u=\frac{\partial^{2} u}{\partial r^{2}}+\frac{1}{r} \frac{\partial u}{\partial r}+\frac{\partial^{2} u}{\partial z^{2}}+\frac{1}{r^{2}} \frac{\partial^{2} u}{\partial \theta^{2}}=-\frac{W(\mathbf{r})}{k^{*}} .
$$

For Dirichlet boundary conditions (i.e., the temperature distribution on the surface is specified), the solution of (3) can be written as

$$
u=y+w,
$$

where

$$
y=y(r, z, \theta)=-k^{*} \oint_{S} u\left(\mathbf{r}^{\prime}\right) \frac{\partial G\left(\mathbf{r} ; \mathbf{r}^{\prime}\right)}{\partial n^{\prime}} d S^{\prime}
$$

and

$$
w=w(r, z, \theta)=\int_{V} W\left(\mathbf{r}^{\prime}\right) G\left(\mathbf{r} ; \mathbf{r}^{\prime}\right) d \tau^{\prime} .
$$

In (5), $n^{\prime}$ is the outward drawn normal to the surface, $d S^{\prime}$ is an element of area on the surface, and the 
integration is taken over the entire surface of the cylinder. In (6), $d \tau^{\prime}$ is an element of volume and the integration is taken over the whole volume of the cylinder. The quantity $G\left(\mathbf{r} ; \mathbf{r}^{\prime}\right)$ is a time-independent Green's function which we choose to express in the two alternative forms $[2,3,4,5,6]$ :

$$
\begin{aligned}
G\left(\mathbf{r} ; \mathbf{r}^{\prime}\right)= & \frac{1}{\pi b k^{*}} \sum_{m=-\infty}^{\infty} \sum_{n=1}^{\infty} \\
& \frac{\cos m\left(\theta-\theta^{\prime}\right) J_{m}\left(\alpha_{m n} r / b\right) J_{m}\left(\alpha_{m n} r^{\prime} / b\right)}{\alpha_{m n} J_{m+1}^{2}\left(\alpha_{m n}\right) \sinh \left(\alpha_{m n} l / b\right)} \\
& \quad \times \sinh \left(\alpha_{m n} \frac{z_{<}}{b}\right) \sinh \left(\alpha_{m n} \frac{l-z_{>}}{b}\right),
\end{aligned}
$$

where $J_{m}$ is the ordinary Bessel function of the first kind and order $m, \alpha_{m n}$ is the $n$th positive root of $J_{m}\left(\alpha_{m n}\right)=0, z_{<}$is the lesser of $z^{\prime}$ or $z$, and $z_{>}$is the greater of $z^{\prime}$ or $z$;

$$
\begin{aligned}
& G\left(\mathbf{r} ; \mathbf{r}^{\prime}\right)=\frac{1}{\pi l k^{*}} \sum_{m=-\infty}^{\infty} \sum_{n=1}^{\infty} \cos m\left(\theta-\theta^{\prime}\right) \\
& \times \quad \sin \left(\frac{n \pi z}{l}\right) \sin \left(\frac{n \pi z^{\prime}}{l}\right) \quad\left[I_{m}\left(\frac{n \pi b}{l}\right) K_{m}\left(\frac{n \pi r_{>}}{l}\right)\right. \\
& \left.-K_{m}\left(\frac{\mathrm{n} \pi b}{l}\right) I_{m}\left(\frac{n \pi r_{>}}{l}\right)\right] \frac{I_{m}\left(n \pi r_{<} / l\right)}{I_{m}(n \pi b / l)},
\end{aligned}
$$

where $I_{m}$ and $K_{m}$ are the modified Bessel functions of the first and second kind, respectively, and order $m, r_{<}$is the lesser of $r^{\prime}$ or $r$, and $r>$ is the greater of $r^{\prime}$ or $r$.

The physical meaning of the two components of (4) is easily seen. If we set $W\left(\mathbf{r}^{\prime}\right)=0$ for all $\mathbf{r}^{\prime}$, we see that $w$ vanishes so that $u=y$. Thus $y$ is the potential distribution due to the boundary conditions in the absence of internal heat generation. If we set $u\left(\mathbf{r}^{\prime}\right)=0$ for all $\mathbf{r}^{\prime}$ on the surface of the cylinder, we see that $y$ vanishes so that $u=w$. Thus $w$ is the potential distribution due to the internal heat generation with the boundaries held at zero potential.

Equations (7) and (8) are completely equivalent; however, in some cases one may be preferred over the other, either because the integrations indicated in (5) or (6) are easier or because the infinite series resulting from these integrations are more rapidly convergent. If the surface integration of (5) or the volume integration of (6) cannot be obtained analytically, $y(\mathbf{r})$ or $w(\mathbf{r})$ can be determined by numerical integration.

Since we are considering the case of a homogeneous, isotropic material, $W\left(\mathbf{r}^{\prime}\right)$ would usually be expected to be axially symmetric for materials which self-heat by radio-active decay. In general $W\left(\mathbf{r}^{\prime}\right)$ would not be axially symmetric for cylinders which are heated by absorption of energy from an external source, e.g., irradiation by neutrons. For the specific case of axial symmetry, we assume the following boundary conditions:

$$
\left.\begin{array}{lll}
z=0 & 0 \leqslant r \leqslant b & u=f(r) \\
z=l & 0 \leqslant r \leqslant b & u=g(r) \\
0 \leqslant z \leqslant l & r=b & u=f(b)+[g(b)-f(b)] \frac{z}{l}+h(z)
\end{array}\right\}
$$

where $f(r)$ and $g(r)$ are arbitrary potential distributions on the flat surfaces of the disk, and where, in order to insure continuity, we shall require that the function $h(z)$ vanishes at the flat surfaces of the disk, so that $h(0)=h(l)=0$. We can substitute these boundary conditions into (5) and obtain

$$
\begin{gathered}
y(r, z)=f(b)+\left[g(b)-f(b) \frac{z}{l}\right] \\
+\sum_{n=1}^{\infty} J_{0}\left(\alpha_{n} \frac{r}{b}\right)\left[A_{n} \sinh \left(\alpha_{n} \frac{z}{b}\right)+B_{n} \sinh \left(\alpha_{n} \frac{l-z}{b}\right)\right] \\
+\sum_{k=1}^{\infty} C_{k} I_{0}\left(\frac{k \pi r}{l}\right) \sin \left(\frac{k \pi z}{l}\right)
\end{gathered}
$$

where $\alpha_{n} \equiv \alpha_{0 n}$ and the coefficients $A_{n}, B_{n}$, and $C_{k}$ are given by

$$
\begin{aligned}
A_{n}=\frac{2}{b^{2} J_{1}^{2}\left(\alpha_{n}\right) \sinh \left(\alpha_{n} l / b\right)} \\
\quad \times \int_{0}^{b}\{g(r)-g(b)\} r J_{0}\left(\alpha_{n} r / b\right) d r,
\end{aligned}
$$

$$
\begin{aligned}
B_{n}=\frac{2}{b^{2} J_{1}^{2}\left(\alpha_{n}\right) \sinh \left(\alpha_{n} l / b\right)} & \\
& \times \int_{0}^{b}\{f(r)-f(b)\} r J_{0}\left(\alpha_{n} r / b\right) d r,
\end{aligned}
$$

and

$C_{k}=\frac{2}{l I_{0}(k \pi b / l)} \int_{0}^{l} h(z) \sin \left(\frac{k \pi z}{l}\right) d z$.

In obtaining (10) from (5), we elected to use (7) to represent $G\left(\mathbf{r} ; \mathbf{r}^{\prime}\right)$ on the flat surfaces of the cylinder and to use (8) on the convex surface. Equation (10) can also be written down without recourse to Green's functions; this was done in [1].

In the specific case of axial symmetry, we can integrate over $\theta$ in (6) and obtain

$w(r, z)=2 \pi \int_{0}^{l} d z^{\prime} \int_{0}^{b} d r^{\prime} r^{\prime} W\left(r^{\prime}, z^{\prime}\right) G\left(r, z ; r^{\prime}, z^{\prime}\right)$,

where the Green's function now takes the two alternative forms: 


$$
\begin{aligned}
G\left(r, z ; r^{\prime}, z^{\prime}\right)= & \frac{1}{\pi b k^{*}} \sum_{n=1}^{\infty} \frac{J_{01}\left(\alpha_{n} r / b\right) J_{0}\left(\alpha_{n} r^{\prime} / b\right)}{\alpha_{n} J_{1}^{2}\left(\alpha_{n}\right) \sinh \left(\alpha_{n} l / b\right)} \\
& \times \sinh \left(\alpha_{n} \frac{z_{<}}{b}\right) \sinh \left(\alpha_{n} \frac{l-z_{>}}{b}\right) ; \\
G\left(r, z ; r^{\prime}, z^{\prime}\right)= & \frac{1}{\pi l k^{*}} \sum_{n=1}^{\infty} \sin \left(\frac{n \pi z}{l}\right) \sin \left(\frac{n \pi z^{\prime}}{l}\right) \\
& \times\left[I_{0}\left(\frac{n \pi b}{l}\right) K_{0}\left(\frac{n \pi r_{>}}{l}\right)\right. \\
- & \left.K_{0}\left(\frac{n \pi b}{l}\right) I_{0}\left(\frac{n \pi r_{>}}{l}\right)\right] \frac{I_{0}\left(n \pi r_{<} / l\right)}{I_{0}(n \pi b / l)} .
\end{aligned}
$$

\subsection{Uniform Heat Generation}

For the special case where $W(\mathbf{r})=W_{0}$, a constant, substituting (15) into (14) and performing the indicated integrations yields

$$
\begin{aligned}
w(r, z)=\frac{2 b^{2} W_{0}}{k^{*}} \sum_{n=1}^{\infty} & \frac{J_{0}\left(\alpha_{n} r / b\right)}{\alpha_{n}^{3} J_{1}\left(\alpha_{n}\right)} \\
& \times\left[1-\frac{\cosh \left[\alpha_{n}(l-2 z) / 2 b\right]}{\cosh \left(\alpha_{n} l / 2 b\right)}\right] .
\end{aligned}
$$

Making use of the identity [7],

$$
8 \sum_{n=1}^{\infty} \frac{J_{0}\left(\alpha_{n} x\right)}{\alpha_{n}^{3} J_{1}\left(\alpha_{n}\right)}=1-x^{2},
$$

eq (17) can be written in the form

$$
\begin{aligned}
& w(r, z)=\frac{b^{2} W_{0}}{4 k^{*}}\left(1-\frac{r^{2}}{b^{2}}\right) \\
& -\frac{2 b^{2} W_{0}}{k^{*}} \sum_{n=1}^{\infty} \frac{J_{0}\left(\alpha_{n} r / b\right)}{\alpha_{n}^{3} J_{1}\left(\alpha_{n}\right)} \frac{\cosh \left[\alpha_{n}(l-2 z) / 2 b\right]}{\cosh \left(\alpha_{n} l / 2 b\right)} .
\end{aligned}
$$

The alternative expression for $w(r, z)$, derived from (16), is

$$
\begin{aligned}
w(r, z)=\frac{4 l^{2} W_{0}}{\pi^{3} k^{*}} \sum_{n=1,3,5 .}^{\infty} \frac{1}{n^{3}} \sin & \left(\frac{n \pi z}{l}\right) \\
\times & {\left[1-\frac{I_{0}(n \pi r / l)}{I_{0}(n \pi b / l)}\right], }
\end{aligned}
$$

where the notation $n=1,3,5$, under the summation sign indicates that the series is to be summed over odd values of $n$ only. Making use of the identity

$$
\frac{8}{\pi^{3}} \sum_{n=1,3,5,}^{\infty} \frac{1}{n^{3}} \sin (n \pi x)=x(1-x),
$$

which can easily be derived, eq (10) can be written in the form

$$
\begin{aligned}
w(r, z)= & \frac{W_{0}}{2 k^{*}} z(l-z) \\
& -\frac{4 l^{2} W_{0}}{\pi^{3} k^{*}} \sum_{n=1,3,5,}^{\infty} \frac{1}{n^{3}} \frac{I_{0}(n \pi r / l)}{I_{0}(n \pi b / l)} \sin \left(\frac{n \pi z}{l}\right) .
\end{aligned}
$$

Equations (19) and (22) could have been derived without recourse to Green's functions for this specific case where $W(\mathbf{r})=W_{0}$ since the leading terms in these two equations are particular solutions to Poisson's equation for this case and are sufficiently simple to be written by inspection. In general, however, this could not be done. In principle, eq (6) provides us with a solution for $w(\mathbf{r})$ regardless of the particular form of $W(\mathbf{r})$ and hence is completely general.

A practical case in which $W(\mathbf{r})$ is essentially constant (and therefore (19) and (22) are applicable) is that of a sample which self-heats by radioactive decay and for which the mean free path of a fission fragment is much less than the dimensions of the sample.

\section{Calculation of Thermal Conductivity}

The total heat flow through a circle of radius $a$, where $a \leqslant b$, at $z=0$ is

$$
\begin{array}{rl}
Q^{\prime \prime}=2 \pi \int_{0}^{a} r k(v)\left(\frac{\partial v}{\partial z}\right)_{z=0} & d r \\
& =2 \pi k^{*} \int_{0}^{a} r\left(\frac{\partial u}{\partial z}\right)_{z=0} d r .
\end{array}
$$

From (4), this can be written as

$$
Q^{\prime \prime}=Q+Q^{\prime},
$$

where

$$
Q=2 \pi k^{*} \int_{0}^{a} r\left(\frac{\partial y}{\partial z}\right)_{z=0} d r=\pi a^{2} k^{*}\left\langle\frac{\partial y}{\partial z}\right\rangle_{z=0}
$$

and

$$
Q^{\prime}=2 \pi k^{*} \int_{0}^{a} r\left(\frac{\partial w}{\partial z}\right)_{z=0} d r=\pi a^{2} k^{*}\left\langle\frac{\partial w}{\partial z}\right\rangle_{z=0} .
$$

From (24), (25), and (26) we obtain

$$
k^{*}=\frac{Q^{\prime \prime}-\pi a^{2} k^{*}\langle\partial w / \partial z\rangle_{z=0}}{\pi a^{2}\langle\partial y / \partial z\rangle_{z=0}} .
$$

This equation gives the thermal conductivity in terms of measurable or calculable quantities. $Q^{\prime \prime}$ is the meas- 
ured total heat flow through the circle $r \leqslant a$ at $z=0$. The quantity $\langle\partial y / \partial z\rangle_{z=0}$, which is the average temperature gradient which would exist over the circle $r \leqslant a$ at $z=0$ due solely to the boundary conditions but with no internal heat generation, can be computed from the observed temperature distribution on the surface of the cylinder using (5) and (25). The quantity $\langle\partial w / \partial z\rangle_{z=0}$. which is the average temperature gradient which would exist over the circle $r \leqslant a$ at $z=0$ due solely to the internal heat generation but with the boundaries maintained at zero potential, can be computed from the (assumed known) rate of heat generation, $W(\mathbf{r})$, in the cylinder using (6) and (26). Note that $w(\mathbf{r})$ is inversely proportional to $k^{*}$ so that the quantity $k^{*}\langle\partial w / \partial z\rangle_{z=0}$ on the right-hand side of (27) is actually independent of $k_{i}^{*}$.

If we again consider the specific case of axial symmetry, (10) can be substituted into (25) to obtain

$$
\begin{aligned}
& Q=\pi a^{2} k^{*}\left[\frac{g(b)-f(b)}{l}\right. \\
&+\frac{2}{a} \sum_{n=1}^{\infty} J_{1}\left(\alpha_{n} \frac{a}{b}\right)\left\{A_{n}-B_{n} \cosh \left(\alpha_{n} \frac{l}{b}\right)\right\} \\
&\left.+\frac{2}{a} \sum_{k=1}^{\infty} C_{k} I_{1}\left(\frac{k \pi a}{l}\right)\right],
\end{aligned}
$$

where $A_{n}, B_{n}$, and $C_{k}$ are given by (11), (12), and (13).

In the case of constant internal heat generation, $W(\mathbf{r})=W_{0}$, and (26) becomes

$$
Q^{\prime}=\pi a^{2} l W_{0} \Omega
$$

where $\Omega=\Omega(a / b ; l / b)$ has two forms corresponding to (19) and (22), respectively:

$$
\begin{aligned}
& \Omega\left(\frac{a}{b} ; \frac{l}{b}\right)=\frac{4 b^{2}}{a l} \sum_{n=1}^{\infty} \frac{J_{1}\left(\alpha_{n} a / b\right)}{\alpha_{n}^{3} J_{1}\left(\alpha_{n}\right)} \tanh \left(\alpha_{n} \frac{l}{2 b}\right), \\
& \Omega\left(\frac{a}{b} ; \frac{l}{b}\right)=\frac{1}{2}-\frac{8 l}{\pi^{3} a} \sum_{n=1,3,5,}^{\infty} \frac{I_{1}(n \pi a / l)}{n^{3} I_{0}(n \pi b / l)} .
\end{aligned}
$$

In the special case, $/ / b \rightarrow 0,(30)$ reduces to

$$
\Omega\left(\frac{a}{b} ; 0\right)=\frac{2 b}{a} \sum_{n=1}^{\infty} \frac{J_{1}\left(\alpha_{n} a / b\right)}{\alpha_{n}^{2} J_{1}\left(\alpha_{n}\right)}=\frac{1}{2},
$$

where we have made use of the identity [8],

$$
4 \sum_{n=1}^{\infty} \frac{J_{1}\left(\alpha_{n} x\right)}{\alpha_{n}^{2} J_{1}\left(\alpha_{n}\right)}=x .
$$

If $l / b \rightarrow 0,(31)$ also reduces to $\Omega=1 / 2$.

In the special case, $a / b \rightarrow 0$, the two forms of $\Omega$ are

$$
\Omega\left(0 ; \frac{l}{b}\right)=\frac{2 b}{l} \sum_{n=1}^{\infty} \frac{\tanh \left(\alpha_{n} l / 2 b\right)}{\alpha_{n}^{2} J_{1}\left(\alpha_{n}\right)}
$$

$$
\Omega\left(0 ; \frac{l}{b}\right)=\frac{1}{2}-\frac{4}{\pi^{2}} \sum_{n=1,3,5,}^{\infty} \frac{1}{n^{2} I_{0}(n \pi b / l)} .
$$

For large values of $/ / b,(30)$ approaches

$$
\Omega=\frac{b}{4 l} \Phi,
$$

where

$$
\Phi=16 \sum_{n=1}^{\infty} \frac{J_{1}\left(\alpha_{n} a / b\right)}{(a / b) \alpha_{n}^{3} J_{1}\left(\alpha_{n}\right)},
$$

Values of $\Phi$ are given in table 3 of [1]. There is an error in table 3 of [1] in the value tabulated for $a / b=0.5$, which should be 1.96630 .

Using a digital computer, numerical values for $\Omega$ have been calculated for a range of values of $a / b$ and $l / b$ and are given in table 1 . Values were calculated using both (30) and (31) and the resultant two sets of values agreed to the number of significant figures given in table 1. Representative values are plotted in figure 1 .

As discussed in [1], the thermal conductivity, $k^{*}$, corresponds to the reference temperature, $T^{*}$, from which the variable, $v$, is measured. In principle, $T^{*}$ may be arbitrarily selected and the mathematics will yield the corresponding value of $k^{*}$. Thermal conductivity values may have to be computed by iteration as discussed in sections 4.2 and 5.2 of [1].

\section{Example}

The above analysis is applicable to the several methods of determining thermal conductivity which

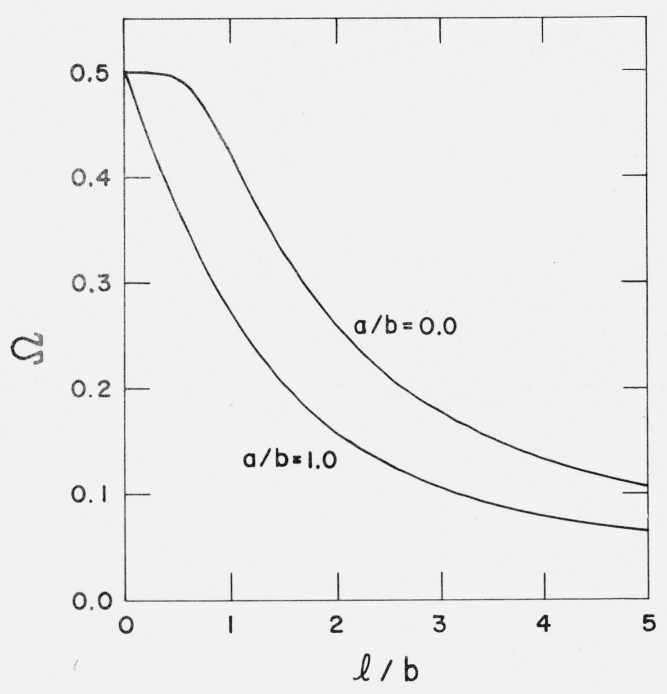

FIGURE 1. The coefficient $\Omega$ for the case of uniform heat generation. The values of $\Omega$ corresponding to intermediate vales of $a / b$ lie between the two curves shown. 
were discussed in [1] and also to other steady-state methods utilizing Dirichlet boundary conditions in which the temperatures at all points on the boundary are specified. In [1], we discussed methods of thermal conductivity determination in which the investigators had assumed parabolic radial potential distributions on the flat surfaces of a cylinder and a linear longitudinal potential on the convex surface. This is a case having radial symmetry so that $(10)$ can be used with the boundary conditions (9), in which $f(r), g(r)$, and $h(z)$ are given by

and

$$
\left.\begin{array}{l}
f(r)=Y_{0}+E_{0} \frac{r^{2}}{b^{2}}, \\
g(r)=Y_{1}+E_{1} \frac{r^{2}}{b^{2}}, \\
h(z)=0
\end{array}\right\}
$$

where $Y_{0}$ and $Y_{1}$ are the potentials (corresponding to $y$ ) at the centers of the faces of the disk, and $E_{0}$ and $E_{1}$ are the potential differences between the edge and the center of the disk at $z=0$ and $z=l$, respectively. For these boundary conditions, (28) becomes

$$
Q=\pi a^{2} \frac{k^{*}}{l}\left[Y_{1}-Y_{0}+E_{1} \Psi_{1}-E_{0} \Psi_{0}\right],
$$

in which

$$
\Psi_{1}=1-16 \sum_{n=1}^{\infty} \frac{J_{1}\left(\alpha_{n} a / b\right)}{(a / b) \alpha_{n}^{3} J_{1}\left(\alpha_{n}\right)} \frac{(l / b)}{\sinh \left(\alpha_{n} l / b\right)}
$$

and

$$
\Psi_{0}=1-16 \sum_{n=1}^{\infty} \frac{J_{1}\left(\alpha_{n} a / b\right)}{(a / b) \alpha_{n}^{3} J_{1}\left(\alpha_{n}\right)} \frac{(l / b)}{\tanh \left(\alpha_{n} l / b\right)}
$$

are factors which depend only on the geometry of the cylinder and on the fraction of the disk from which the heat flow is measured. Numerical values of $\Psi_{0}$ and $\Psi_{1}$ are given in tables 1 and 2 of [1] for a range of values of $a / b$ and $l / b$.

In [1] we did not discuss the effect of internal heat generation. For the special case of parabolic radial potential distributions on the flat surface of the cylinder, a linear longitudinal potential on the convex

\begin{tabular}{|c|c|c|c|c|c|c|c|c|c|c|c|c|}
\hline & 0.0 & 0.10 & 0.20 & 0.30 & 0.40 & 0.50 & 0.60 & 0.70 & 0.80 & 0.90 & 1.00 & \\
\hline 0.0 & 0.50000 & 0.50000 & 0.50000 & 0.50000 & 0.50000 & 0.50000 & 0.50000 & 0.50000 & 0.50000 & 0.50000 & 0.50000 & 0.0 \\
\hline .1 & .50000 & .50000 & .50000 & .50000 & .50000 & .50000 & .50000 & .50000 & .49993 & .49872 & .47328 & .1 \\
\hline .2 & .50000 & .50000 & .50000 & .50000 & .49998 & .49995 & .49980 & .49924 & .49700 & .48786 & .44742 & .2 \\
\hline .3 & .49991 & 49989 & 49985 & 49974 & .49949 & .49894 & .49766 & .49466 & .48745 & .46973 & .42244 & .3 \\
\hline .4 & .49891 & .49883 & .49854 & .49796 & .49689 & .49495 & .49138 & .48477 & .47234 & .44841 & .39836 & .4 \\
\hline 0.5 & .49535 & .49511 & .49437 & .49295 & .49057 & .48670 & .48044 & .47030 & .45368 & .42584 & .37523 & 0.5 \\
\hline .6 & .48796 & .48754 & .48623 & .48384 & .48003 & .47423 & .46554 & .45257 & .43303 & .40301 & .35309 & $\begin{array}{l}0.0 \\
.6\end{array}$ \\
\hline .7 & .47657 & .47597 & .47413 & .47084 & .46577 & .45836 & .44779 & .43281 & .41148 & .38051 & .33201 & .7 \\
\hline .8 & .46181 & .46107 & .45878 & .45478 & .44873 & .44015 & .42828 & .41205 & .38980 & .35872 & .31205 & .8 \\
\hline .9 & .44463 & .44378 & .44118 & .43666 & .42995 & .42059 & .40793 & .39105 & .36851 & .33789 & .29327 & .9 \\
\hline 1.0 & .42599 & .42507 & .42226 & .41742 & .41030 & .40050 & .38747 & .37038 & .34800 & .31819 & .27570 & 1.0 \\
\hline 1.2 & .38740 & .38643 & .38346 & .37840 & .37103 & .36108 & .34809 & .33144 & .31014 & .28250 & .24421 & 1.2 \\
\hline 1.4 & .35038 & .34942 & .34651 & .34156 & .33441 & .32483 & .31247 & .29681 & .27706 & .25180 & .21737 & 1.4 \\
\hline 1.6 & .31690 & .31598 & .31322 & .30853 & .30180 & .29282 & .28130 & .26683 & .24870 & .22571 & .19469 & 1.6 \\
\hline 1.8 & .28749 & .28664 & .28406 & .27969 & .27343 & .26511 & .25448 & .24117 & .22459 & .20366 & .17558 & 1.8 \\
\hline 2.0 & .26202 & .26123 & .25884 & .25479 & .24900 & .24132 & .23153 & .21930 & .20411 & .18499 & .15944 & 2.0 \\
\hline 2.2 & .24006 & .23932 & .23711 & .23337 & .22802 & .22093 & .21190 & .20064 & .18668 & .16914 & .14575 & 2.2 \\
\hline 2.4 & .22111 & .22043 & .21838 & .21491 & .20996 & .20339 & .19505 & .18465 & .17176 & .15559 & .13406 & 2.4 \\
\hline 2.6 & .20471 & .20408 & .20217 & 19895 & .19435 & .18825 & .18050 & .17086 & .15891 & .14394 & .12401 & 2.6 \\
\hline 2.8 & .19044 & 18985 & .18807 & .18506 & .18077 & .17509 & .16788 & .15889 & .14777 & .13384 & .11530 & 2.8 \\
\hline 3.0 & .17794 & .17739 & .17573 & .17292 & .16890 & .16359 & .15684 & .14844 & .13804 & .12502 & .10770 & 3.0 \\
\hline 3.5 & .15272 & .15224 & .15081 & .14840 & .14495 & .14038 & .13458 & .12737 & .11844 & .10726 & .09240 & 3.5 \\
\hline 4.0 & .13368 & .13326 & .13201 & . 12990 & .12687 & .12288 & .11780 & .11148 & .10367 & .09388 & .08088 & 4.0 \\
\hline 4.5 & .11884 & .11847 & .11736 & .11548 & .11279 & .10923 & .10472 & .09910 & .09216 & .08346 & .07190 & 4.5 \\
\hline \multirow[t]{2}{*}{5.0} & .10696 & .10663 & .10562 & 10393 & .10151 & .09831 & .09425 & .08920 & .08294 & .07511 & .06471 & 5.0 \\
\hline & 0.0 & 0.10 & 0.20 & 0.30 & 0.40 & 0.50 & 0.60 & 0.70 & 0.80 & 0.90 & 1.00 & \\
\hline
\end{tabular}
surface, and uniform heat generation throughout the cylinder, (27) becomes

TABLE 1. The coefficient $\Omega$ as a function of $\mathrm{l} / \mathrm{b}$ and $\mathrm{a} / \mathrm{b}$ for uniform heat generation 


$$
k^{*}=\frac{Q^{\prime \prime}-\pi a^{2} l W_{0} \Omega}{\left(\pi a^{2} / l\right)\left[Y_{1}-Y_{0}+E_{1} \Psi_{1}-E_{0} \Psi_{0}\right]} .
$$

As a specific example of the use of (42), we consider the method described by Hoch et al. [9], in which the heat flux from the flat surfaces of the disk is calculated from the temperature and the total hemispherical emittance of the specimen surface, using the StefanBoltzmann radiation law. That is, the heat flow, $Q^{\prime \prime}$, is given by

$$
Q^{\prime \prime}=\pi a^{2} \epsilon \sigma \overline{T^{4}}
$$

where $\epsilon$ is the total hemispherical emittance, $\sigma$ is the Stefan-Boltzmann constant, and $\overline{T^{4}}$ is the average value of $T^{4}$ on the surface over the circle of radius $a$. Substitution of (43) into (42) results in

$$
k^{*}=\frac{l\left[\epsilon \sigma \overline{T^{4}}-l W_{0} \Omega\right]}{Y_{1}-Y_{0}+E_{1} \Psi_{1}-E_{0} \Psi_{0}} .
$$

With the simplified boundary conditions assumed by Hoch et al., namely $Y_{1}=Y_{0}$ and $E_{1}=E_{0}$, this reduces to

$$
k^{*}=\frac{l\left[\epsilon \sigma \overline{T^{4}}-l W_{0} \Omega\right\rfloor}{E_{0}\left[\Psi_{1}-\Psi_{0}\right\rfloor} .
$$

If this is evaluated at the center of the disk (i.e., $a=0$ ), as was done by Hoch et al., the thermal conductivity is given by

$$
k^{*}=\frac{l\left[\epsilon \sigma T_{0}^{4}-l W_{0} \Omega\right\rfloor}{E_{0}\left[\Psi_{1}-\Psi_{0}\right]},
$$

where $T_{0}$ is the (absolute) temperature at the center of the disk at $z=0$, and $\Psi_{0}, \Psi_{1}$ and $\Omega$ are to be evaluated at $a / b=0$. If there is no heat generation (i.e., $W_{0}=0$ ), equation (46) reduces to a form which is exactly equivalent to eq (13) of Hoch et al. [9].

We postulate the following data as having been acquired experimentally:

$$
\begin{array}{rlrl}
b & =1.00 \mathrm{~cm} & l & =1.80 \mathrm{~cm} \\
\epsilon & =0.17 & W_{0} & =14.3 \mathrm{~W} / \mathrm{cm}^{3} \\
T_{0} & =2360{ }^{\circ} \mathrm{K} & E_{0} & =28 \mathrm{deg} .
\end{array}
$$

From tables 1 and 2 of [1] for the case $a / b=0.0$ and $l / b=1.8$, we obtain

$$
\Psi_{1}=0.87364 \quad \Psi_{0}=-2.85220 .
$$

From table 1 of the present paper, we obtain

$$
\Omega=0.28749 .
$$

Using $\sigma=5.6697 \times 10^{-12} \mathrm{~W} / \mathrm{cm}^{2} \mathrm{deg}^{4}$, substitution of the above values into (46) yields

$$
k^{*}=0.388 \mathrm{~W} / \mathrm{cm} \mathrm{deg}
$$

as the thermal conductivity of the sample at the temperature corresponding to $v=0$.

\section{References}

[1] D. R. Flynn, Heat flow in a right circular cylinder with arbitrary temperature boundary conditions - applications to the determination of thermal conductivity, J. Res. NBS 68C (Eng. and Instr.), No. 4, 215 (1964).

[2] A. Gray and G. B. Mathews, A Treatise on Bessel Functions and Their Applications to Physics, 2d ed., p. 107 (MacMillan, 1922). Also available as a reprint edition (Dover, 1966).

[3| P. M. Morse and H. Feshback, Methods of Theoretical Physics, Part II (McGraw-Hill Book Co., New York, N.Y., 1953).

[4] S. Bergman and M. Schiffer, Kernel Functions and Elliptic Differential Equations in Mathematical Physics (Academic Press, 1953).

[5] H. S. Carslaw and J. C. Jaeger, The Conduction of Heat in Solids, 2d Edition (Oxford Univ. Press, 1959).

[6] J. D. Jackson, Classical Electrodynamics (John Wiley \& Sons, New York, N.Y., 1962).

[7] L. F. Epstein, Some infinite sums involving zeros of $J_{0}(x)$, J. Res. NBS 68B (Math. and Math. Phys.) No. 1, 20, eq (3-2), (1964).

[8] Ibid, p. 20, second equation after (3-1).

[9] M. Hoch, D. A. Nitti, C. F. Gottschlich, and P. E. Blackburn, New method for the determination of thermal conductivities between 1000 and $3000{ }^{\circ} \mathrm{C}$, in Progress in International Research on Thermodynamic and Transport Properties, p. 512 (ASME, Academic Press, 1962).

(Paper 71C4-260) 